\title{
Defibrillation testing during implantation of the subcutaneous implantable cardioverter-defibrillator: a necessary standard or becoming redundant?
}

\author{
W. van der Stuijt · A. B. E. Quast · R. E. Knops
}

(C) The Author(s) 2020

\begin{abstract}
Since the publication of the SIMPLE and NORDIC trials, defibrillation testing (DFT) is rarely performed during routine implantation of transvenous implantable cardioverter-defibrillators (ICD). However, the results of these trials cannot be extrapolated to the later introduced subcutaneous ICD (SICD) and a class I recommendation to perform DFT during the implantation of these devices remains in the current guidelines. Due to the high conversion success rate of DFT on one hand, and the risk of complications on the other, a significant number of physicians omit DFT in S-ICD recipients. Several retrospective analyses have assessed the safety of the omission of DFT and report contradicting results and recommendations. It is known that implant position, as well as device factors and patient characteristics, influence defibrillation success. A better comprehension of these factors and their relationship could lead to more reliable and safer alternatives to DFT. An ongoing randomised clinical trial, which is expected to end in 2023, is the first study to implement a method that assesses implant position to identify patients who are likely to fail their DFT.
\end{abstract}

Keywords Implantable defibrillator · Defibrillation testing · Safety margin testing $\cdot$ Ventricular fibrillation · Sudden cardiac death

\section{Introduction}

The implantable cardioverter-defibrillator (ICD) is standard of care for patients with potentially lifethreatening ventricular arrhythmias and those at high

W. van der Stuijt $(\bowtie)$ · A. B. E. Quast · R. E. Knops Department of Cardiology, Heart Center, Amsterdam Cardiovascular Sciences, Amsterdam UMC, University of Amsterdam, Amsterdam, The Netherlands

w.vanderstuijt@amsterdamumc.nl risk of sudden cardiac death [1,2]. Defibrillation testing (DFT) has traditionally been part of the implant procedure of the ICD to confirm adequate defibrillation and appropriate sensitivity of the device. This used to be performed through step-down protocols determining the actual defibrillation threshold, and in the beginning these tests were even periodically repeated to ensure an appropriate safety margin. This has evolved into the common clinical practice where a single shock conversion test is performed to ensure adequate device functionality. In transvenous ICDs, adhering to a safety margin of $10 \mathrm{~J}$ is highly recommended, although physicians may deviate from this practical guideline in certain cases. The most accepted definition defines success of DFT as an ICD shock that successfully terminates an induced ventricular arrhythmia. The landmark trials SIMPLE and NORDIC [3, 4] demonstrated omission of routine DFT to be non-inferior to standard of care with regard to arrhythmic death and first shock efficacy in transvenous ICDs. Although the non-inferiority margin in both studies was quite different, both conclude that a no testing strategy should be preferred because of the risk of complications and lack of benefit from DFT. The omission of routine DFT during routine implantation of left-sided transvenous ICDs is since included in current guidelines with a class IIa recommendation. Except for certain patient populations which are considered to have a high risk of an elevated defibrillation threshold, DFT is now rarely performed. Implantations during which DFT is still recommended include patients with hypertrophic cardiomyopathy or arrhythmogenic right ventricular cardiomyopathy, but also right-sided transvenous ICD implantations.

As the subcutaneous ICD (S-ICD) has become a safe and feasible alternative to the transvenous ICD, the issues of routinely performing DFT during implantation have once again become an issue. The results 
Table 1 Overview of the current guidelines on defibrillation testing

\begin{tabular}{|c|c|}
\hline \multicolumn{2}{|c|}{2015 HRS/EHRA/APHRS/SOLAECE expert consensus statement } \\
\hline Class I & Defibrillation efficacy testing is recommended in patients undergoing a subcutaneous ICD implantation (level C) \\
\hline Class lla & $\begin{array}{l}\text { It is reasonable to omit defibrillation efficacy testing in patients undergoing initial left pectoral transvenous ICD implantation procedures } \\
\text { where appropriate sensing, pacing, and impedance values are obtained with fluoroscopically well-positioned RV leads (level B) } \\
\text { Defibrillation efficacy testing is reasonable in patients undergoing right pectoral transvenous ICD implantation or ICD pulse generator } \\
\text { changes (level B) }\end{array}$ \\
\hline Class III & $\begin{array}{l}\text { Defibrillation efficacy testing at time of implantation of a transvenous ICD should not be performed on patients with a documented } \\
\text { non-chronic cardiac thrombus, atrial fibrillation or atrial flutter without adequate systemic anticoagulation, critical aortic stenosis, unstable } \\
\text { CAD, recent stroke or TIA, haemodynamic instability, or other known comorbidities associated with poor outcomes (level C) }\end{array}$ \\
\hline \multicolumn{2}{|c|}{ Boston Scientific Manual Emblem MRI } \\
\hline Class Illb & Defibrillation testing may be conducted with a recommended 15J safety margin \\
\hline
\end{tabular}

of the abovementioned landmark trials cannot simply be extrapolated to the later introduced S-ICD. In the SIMPLE trial, patients were only randomised to either DFT strategy when adequate positioning of the device was confirmed, which was done by adequate sensory signals, an R-wave of at least $5.0 \mathrm{mV}$, and an acceptable high-voltage impedance. These measurements established appropriate sensing of the transvenous device and functioned as a safety net whilst omitting DFT in patients randomised to this strategy. However, the sensing characteristics of the S-ICD are completely modified because of its extrathoracic position. While the intracardiac signal of a transvenous system resembles an intracardiac electrogram, the subcutaneous signal is similar to the surface electrocardiogram. This morphology-based classification only uses one vector, as opposed to the bipolar sensing or dualcoil the transvenous ICD uses to verify the ventricular arrhythmia. This makes the S-ICD more prone to oversensing of noise and $\mathrm{T}$ waves, but also results in less suitable measurements to confirm appropriate device positioning during implantation. Moreover, as the S-ICD is implanted using anatomical landmarks, there is a wide range in possible implantation positions, which affects the ability of the device to defibrillate successfully. Therefore, there is still a class I recommendation to perform DFT during the implantation of the S-ICD (Tab. 1). In this review, we aim to elaborate on the potential benefits and disadvantages of DFT, the factors contributing to defibrillation success in S-ICD implants and the future perspectives on this topic.

\section{Rationale of routine DFT during S-ICD implantation}

The performance of DFT during S-ICD implantation is recommended to confirm system integrity and reliable sensing. First, for unexperienced implanters, performing DFT is a quick and straightforward method to ensure device positioning and functionality. Furthermore, technical issues where the device fails to sense ventricular fibrillation (VF) are difficult to recognise before or during implantation without DFT, especially with the limited options to confirm the sensing in the S-ICD. The shock vector varies with the implant posi- tion, and the implanter has a wide range in possible implant positions. Finally, in patients at risk for a high defibrillation threshold, such as those who take amiodarone, or who are diagnosed with hypertrophic cardiomyopathy or arrhythmogenic right ventricular cardiomyopathy, performing DFT may be the only way to guarantee an appropriate safety margin.

\section{Disadvantages of DFT in S-ICD recipients}

It may be undesirable to expose all patients to DFT, since the induction of VF and subsequent defibrillation is associated with various complications, albeit with a low incidence rate [5]. The most common complication associated with the performance of DFT is prolonged resuscitation, which in some cases even results in death. However, also complications related to the required anaesthesia, as well as cerebral stroke and inability to convert, have been reported. Haemodynamically comprising complications are more prevalent in patients with a left ventricular function $<30 \%$, which is the case for most cardiovascular ICD patients [6, 7]. Moreover, several studies found that defibrillation shocks may cause myocardial damage, depicted as transient depression of left ventricular function, prolonged asystole and an increase in serum troponin levels [8-14]. The latter, however, may be associated with the active fixation

\section{Dutch contribution to the field}

- Amsterdam UMC, location AMC is the sponsor of the PRAETORIAN-DFT trial, which is an international multicentre trial.

- The PRAETORIAN score and the computer modelling study it was designed after were both a production of a Dutch S-ICD research group.

- The finding that troponin release after defibrillation testing in transvenous ICDs is associated with active lead fixation instead of the defibrillation shocks was first reported by a Dutch S-ICD research group.

- Step-down defibrillation testing in the S-ICD was a publication by a Dutch S-ICD research group. 
Fig. 1 Delivered energy versus probability of successful defibrillation in the S-ICD

Fig. 2 The success-rate of DFT is unpredictable and depends on the amount of tests
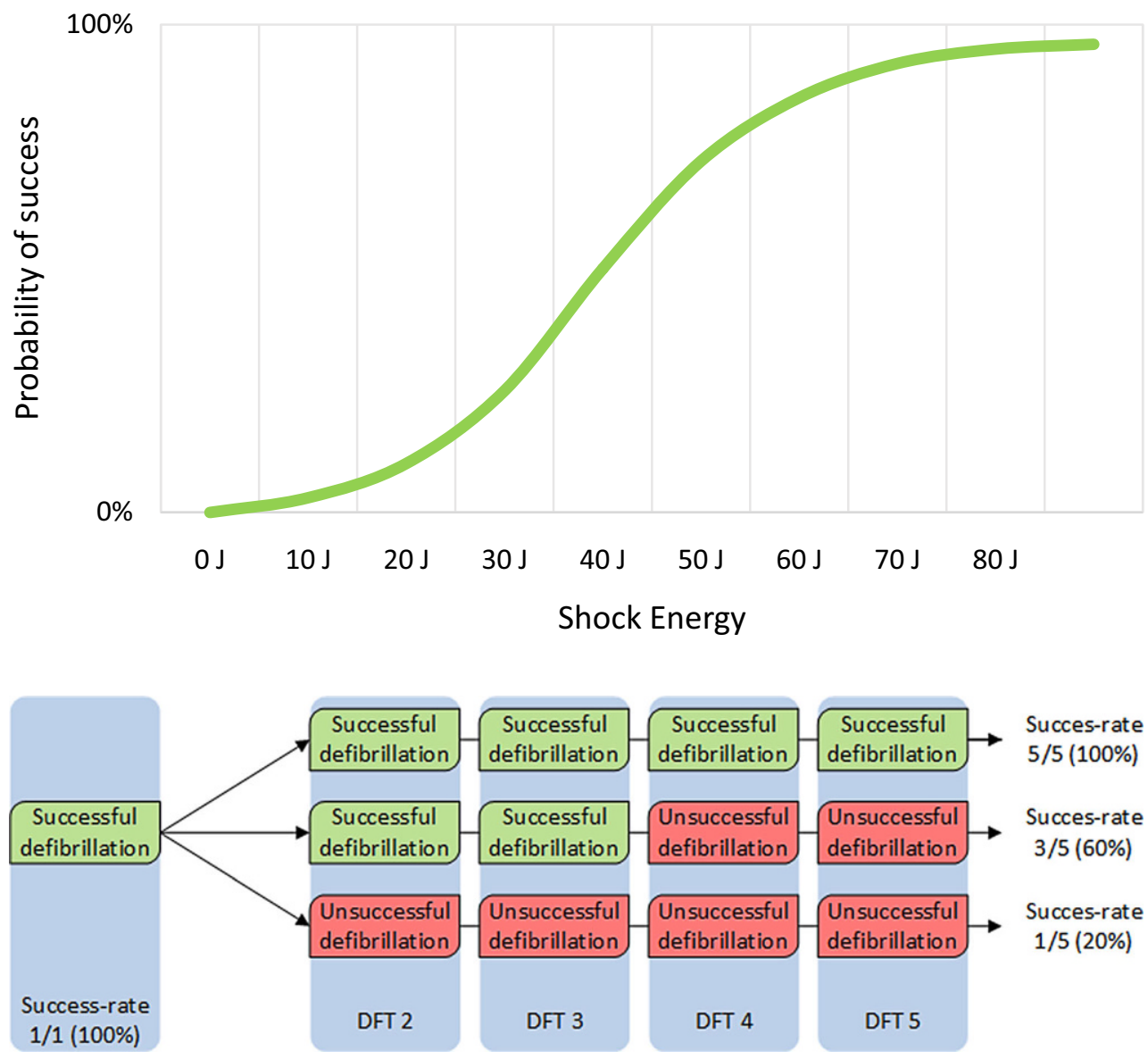

of the endocardial lead rather than the shock itself [15]. Finally, the extra personnel required to perform DFT poses a logistical and financial burden on our healthcare system.

Another disadvantage of DFT is the difficult interpretation of the results. There is no threshold in DFT where all shocks above are successful and all shocks below fail. Defibrillation is therefore often described by a probability-of-success curve. The probability of success at a certain shock energy is estimated by the result of a series of defibrillation tests at that same shock energy. This is then repeated at different shock outputs, resulting in a dose-response probability curve, which differs per patient (Fig. 1). Additionally, when a defibrillation shock fails, it is unpredictable whether the next shock at the same shock output will fail too, making the outcome of DFT difficult to appreciate (Fig. 2).

\section{Comprehension of defibrillation success}

Although the defibrillation dose is generally evaluated by the delivered energy of the defibrillator, defibrillation success relies on the amount of electrical current that passes through the myocardium.

This electrical current-and thus defibrillation success-is dependent on several patient characteristics, implant factors and device factors, which also influence each other (Fig. 3). When we acknowledge and understand all these factors, we might be able to safely omit DFT or even predict defibrillation success without testing in the future. Although various factors remain currently unidentified, we describe five factors that are known to influence defibrillation success.

1. Shock output

The electrical current is affected by the shock output of the device. The standard shock output of the S-ICD is based on the results of DFTs in two pilot studies with temporarily implanted S-ICDs following a step-down protocol. They aimed to assess the defibrillation threshold, i.e. the minimum shock output that effectively terminated the induced ventricular arrhythmia. The first study enrolled 78 patients and reported an average defibrillation threshold of $32.5 \pm 17.0 \mathrm{~J}$ and the second study reported an average defibrillation threshold of $36.6 \pm 19.8 \mathrm{~J}$ after studying 49 patients [16]. With a safety margin of 15J, a shock output of $80 \mathrm{~J}$ is deemed safe and effective.

2. Implant position

Recently, a computer model study identified three factors that alter the defibrillation threshold in S-ICD patients: 1) fat tissue underneath the coil, 
2) fat tissue underneath the generator and 3) an anterior placement of the generator on the thoracic wall [17]. When implanting physicians alter their implanting technique with these aspects in mind, step-down testing showed a lower average defibrillation threshold [18]. When implanted intermuscularly with subsequently no fat tissue underneath the generator, another study demonstrated a successful DFT at $\leq 40 \mathrm{~J}$ in $94 \%$ of the patients [19].

3. Impedance

The current flow is determined not only by the selected shock output, but also by the resistance of the human body. The resistance of the human body between the coil and generator during a shock is represented by the high voltage impedance. As such, patients with high impedance during DFT have a greater chance at DFT failure. However, neither the range nor the determining factors of high voltage impedance of the S-ICD have been properly evaluated in humans.

4. Patient characteristics

Case reports suggest that the variance in tissue with poor conductive properties, such as fat tissue or emphysematous lung tissue, could explain the range of impedance [20, 21]. Large chest circumference is also a determinant of DFT failure, but this is perhaps solely a consequence of the amount of nonconductive pneumatic tissue [22]. Moreover, cardiac mass and septum thickness are also known to influence defibrillation threshold and defibrillation success [23]. A high body mass index is also associated with a higher risk of defibrillation failure, but this could be related to an inferior implant position with fat tissue underneath the lead or generator.

5. Device design

Device factors also contribute to successful defibrillation. In automatic external defibrillators, it is known that a larger paddle size results in a lower shock impedance, probably resulting in a higher shock efficacy [22]. This could mean that a larger generator or lead surface may also result in a higher shock efficacy, but clinical studies testing this hypothesis are lacking.

\section{Omission of DFT in S-ICD implants}

Regardless of the strong recommendation in the current guidelines, a recent study showed that DFT is only performed in $75 \%$ of the patients undergoing de novo S-ICD implantation in the United States [24]. This low rate is explained by the suggestion that physicians may omit DFT in high-risk patients due to the risk of complications, while simultaneously being resilient with DFT in patients who are likely to have a successful test. No randomised clinical trials have assessed the safety of omitting DFT in S-ICD recipients yet, but some retrospective analyses have shown diverse outcomes. One analysis showed no difference in mortality between the S-ICD and transvenous ICD when

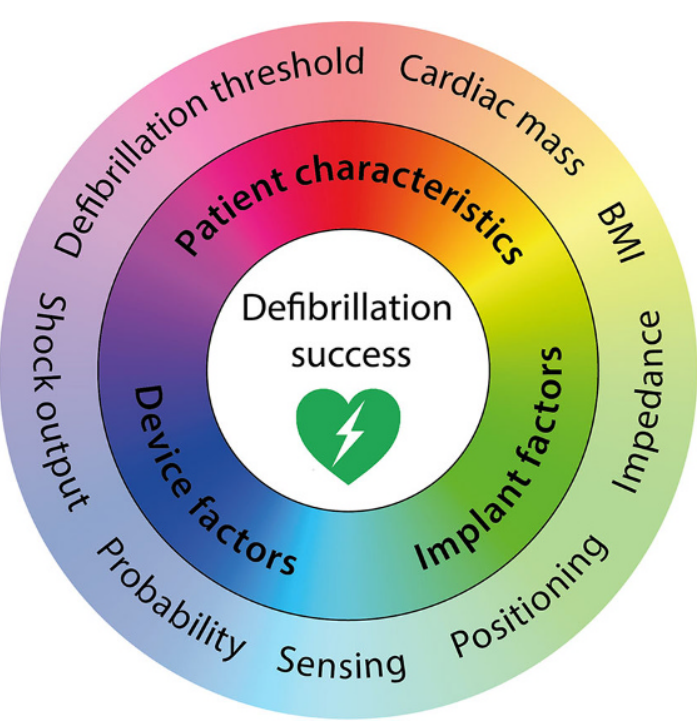

Fig. 3 Defibrillation success depends on patients characteristics, device factors and implant factors

no DFT was performed during implant [25]. A retrospective comparison between S-ICD with and without DFT during implant showed no effect on first shock efficacy of the device[26] and a third analysis reported successful defibrillation of all spontaneous ventricular arrhythmias $(n=6)$ after no DFT was performed during implant [27]. On the other hand, one study reported sustained noise oversensing during induced VF in $4 \%$ of S-ICD patients, which resulted in delay of therapy,[28] although this could be the result of oversensing of myopotentials by the diaphragmatic muscles caused by the induction of VF [29]. Another single centre study showed that the first shock during DFT was successful in just $75 \%$ and argues that DFT is still necessary for S-ICD implants [30]. However, these results do not comply with the first published studies about S-ICD safety, which showed a high conversion success rate of DFT during S-ICD implants, varying from $98.7-100 \%$ [31-34]. Tab. 2 shows an overview of the DFT success rate and first shock efficacy in spontaneous events of the S-ICD, as reported in recent literature.

At present, physicians are exploring alternatives to avoid the complications associated with DFT. Some prefer impedance measurements, because the high voltage impedance can be measured with a synchronous 10J shock and without the need for VF induction. However, a low shock impedance may be the result of shunting over the thoracic wall, with no electrical current passing through the myocardium. Therefore, low impedance does not guarantee successful defibrillation, making it unsuited as a surrogate for DFT. Others came up with a method to assess implant position to identify patients who are likely to fail their DFT [35]. Currently, an ongoing randomised controlled trial is testing the hypothesis that S-ICD implantation is safe when these implant requirements 
Table 2 Literature overview of DFT success rate and first shock efficacy in spontaneous events of the S-ICD

\begin{tabular}{l}
$\begin{array}{l}\text { Author [year of publica- } \\
\text { tion] (ref) }\end{array}$ \\
Weiss [2013] [34] \\
Frommeyer [2016] [30] \\
Maurizi [2017] [26] \\
Boersma [2017] [31] \\
Gold [2017] [33] \\
\hline Peddareddy [2018] [27] \\
Le Polain de Waroux [2018] [28] \\
Boersma [2019] [32]
\end{tabular}

DFT success rate

$100 \%(304 / 304)$

$75 \%(74 / 98)$

$97.6 \%(40 / 41)$

$99.5 \%(857 / 861)$

$98.7 \%(1394 / 1412)$

$83.7 \%(113 / 135)$

$92 \%(118 / 128)$

$99.2 \%(905 / 912)$

\author{
First shock efficacy in spontaneous \\ events \\ $92.1 \%(35 / 38)$ \\ NA \\ NA \\ 88.5\% Unknown \\ NA \\ $88.4 \%(61 / 69)$ \\ NA \\ NA
}

are met: PRAETORIAN-DFT (NCT03495297) [36]. This trial, the results of which are expected in 2023, will be the first study to take the different factors that influence defibrillation success into account.

\section{Considerations for DFT in special populations}

After publication of PRAETORIAN-DFT, it could be that routine DFT in de novo S-ICD implantations will no longer be recommended by the guidelines. However, in certain populations it remains rational to perform DFT to ensure device functionality. Confirmation of appropriate sensing is especially required in patients whose QRS amplitude has diminished after initial implant and as a consequence are at risk of undersensing of VF, or those who have experienced failed appropriate shocks. Furthermore, in patients with a deviant chest anatomy, such as pectus carinatum, the defibrillation threshold is unpredictable. In morbidly obese patients (body mass index $>35 \mathrm{~kg} / \mathrm{m}^{2}$ ), there is a higher chance of an inferior implanting position, especially in unexperienced implanters. Although DFT could help to assess the implant position and chance of successful defibrillation, less invasive methods are preferred. Finally, randomised clinical trials in paediatric patients are extremely rare, and it is unlikely that omission of DFT during S-ICD implant in children will find any scientific basis. However, one could argue that the shock output of 80J combined with the low body mass of children will almost always result in successful defibrillation, possibly making DFT redundant.

\section{Conclusions}

Despite a class I recommendation, many physicians decide to omit DFT in S-ICD recipients. Indeed, DFT comes with a risk of various complications and the results are difficult to interpret due to probability. With a more thorough comprehension of the patient characteristics, implant factors and device factors that influence defibrillation success, we may be able to safely omit DFT during S-ICD implants in the future. An ongoing randomised clinical trial, which is expected to end in 2023, is the first study that implements a method that assesses implant position to identify patients who are likely to fail their DFT.

Conflict of interest W. van der Stuijt and A.B.E. Quast have nothing to disclose. R.E. Knops reports consultancy fees and research grants from Abbott, Boston Scientific, Cairdac and Medtronic, as well as stock options from AtaCor Medical Inc.

Open Access This article is licensed under a Creative Commons Attribution 4.0 International License, which permits use, sharing, adaptation, distribution and reproduction in any medium or format, as long as you give appropriate credit to the original author(s) and the source, provide a link to the Creative Commons licence, and indicate if changes were made. The images or other third party material in this article are included in the article's Creative Commons licence, unless indicated otherwise in a credit line to the material. If material is not included in the article's Creative Commons licence and your intended use is not permitted by statutory regulation or exceeds the permitted use, you will need to obtain permission directly from the copyright holder. To view a copy of this licence, visit http://creativecommons.org/licenses/by/4.0/.

\section{References}

1. Al-Khatib SM, Stevenson WG, Ackerman MJ, et al. 2017 AHA/ACC/HRS guideline for management of patients with ventricular arrhythmias and the prevention of sudden cardiac death: a report of the American College of Cardiology/ American Heart Association Task Force on Clinical Practice Guidelines and the Heart Rhythm Society. Heart Rhythm. 2018;15:e73-e189.

2. Priori SG, Blomstrom-Lundqvist C, Mazzanti A, et al. 2015 ESC Guidelines for the management of patients with ventricular arrhythmias and the prevention of sudden cardiac death: The Task Force for the Management of Patients with Ventricular Arrhythmias and the Prevention of Sudden Cardiac Death of the European Society of Cardiology (ESC) endorsed by: Association for European Paediatric and CongenitalCardiology (AEPC).Europace. 2015;17:1601-87.

3. Bansch D, Bonnemeier $\mathrm{H}$, Brandt J, et al. Intra-operative defibrillation testing and clinical shock efficacy in patients with implantable cardioverter-defibrillators: the NORDIC ICD randomized clinical trial. Eur Heart J. 2015;36:2500-7.

4. Healey JS, Hohnloser SH, Glikson M, et al. Cardioverter defibrillator implantation without induction of ventricular fibrillation: a single-blind, non-inferiority, randomised controlled trial (SIMPLE). Lancet. 2015;385:785-91.

5. Birnie D, Tung S, Simpson C, et al. Complications associated with defibrillation threshold testing: the Canadian experience. Heart Rhythm. 2008;5:387-90. 
6. Kim SG, Fisher JD, Choue CW, et al. Influence of left ventricular function on outcome of patients treated with implantable defibrillators. Circulation. 1992;85:1304-10.

7. Steinbeck G, Dorwarth U, Mattke S, et al. Hemodynamic deterioration during ICD implant: predictors of high-risk patients. Am Heart J. 1994;127(4 Pt2):1064-7.

8. D'Onofrio A, Russo V, Bianchi V, etal. Effects of defibrillation shock in patients implanted with a subcutaneous defibrillator: a biomarker study. Europace. 2018;20(FI2):f233-f9.

9. Francis CK, Kuo YH, Azzam I, et al. Brain natriuretic peptide and biomarkers of myocardial ischemia increase after defibrillation threshold testing. Pacing Clin Electrophysiol. 2012;35:314-9.

10. Garcia R, Inal S, Favreau F, et al. Subcutaneous cardioverter defibrillator has longer time to therapy but is less cardiotoxic than transvenous cardioverter defibrillator. Study carried out in a preclinical porcine model. Europace. 2018;20:873-9.

11. Ishigaki D, Kutsuzawa D, Arimoto T, et al. The association between defibrillation shockenergy and acutecardiac damage in patients with implantable cardioverter defibrillators. JArrhythm. 2016;32:481-5.

12. Killingsworth CR, Melnick SB, Litovsky SH, Ideker RE, Walcott GP. Evaluation of acute cardiac and chest wall damage after shocks with a subcutaneous implantable cardioverter defibrillator in Swine. Pacing Clin Electrophysiol. 2013;36:1265-72.

13. Semmler V, Biermann J, Haller B, et al. ICD shock, not ventricular fibrillation, causes elevation of high sensitive troponin $\mathrm{T}$ after defibrillation threshold testing-the prospective, randomized, multicentre tropshock-trial. PLoS ONE. 2015;10:e131570.

14. Vamos M, Healey JS, Wang J, et al. Troponin levels after ICD implantation with and without defibrillation testing and their predictive value for outcomes: Insights from the SIMPLE trial. Heart Rhythm. 2016;13:504-10.

15. Quast ABE, Baalman SWE, Van der Stuijt W, et al. Rapid fire 4: the shocking truth of device therapy. Abstract $312 \mathrm{My}-$ ocardial damage as a result of implantation and defibrillation testing in transvenous and subcutaneous implantable cardioverter-defibrillators. Europace. 2019;21(Suppl 2):ii940-ii51.

16. Bardy GH, Smith WM, Hood MA, et al. An entirely subcutaneous implantable cardioverter-defibrillator. NEngl J Med. 2010;363:36-44.

17. Heist EK, Belalcazar A, Stahl W, Brouwer TF, Knops RE. Determinants of subcutaneous Implantable cardioverterdefibrillator efficacy: a computer modeling study. JACC Clin Electrophysiol. 2017;3:405-14.

18. Quast ABE, Baalman SWE, Van der Stuijt W, Wilde AAM, Knops RE. Minimal defibrillation thresholds and the correlation with implant position in subcutaneous implantable-defibrillator patients. J Cardiovasc Electrophysiol. 2019;30:2441-7.

19. BiffiM,Ziacchi M, AngelettiA, etal. Successful defibrillation verification in subcutaneous implantable cardioverter-defibrillator recipients by low-energy shocks. Clin Cardiol. 2019;42:612-7.

20. Cheung CC, Larsen JM, Bashir J, Dorian P, Laksman ZW. The potential impact of Intrathoracic impedance on defibrillation threshold testing in S-ICDs. Can J Cardiol. 2019;35:1604.e13-1604.e16.

21. Frankel DS, Burke MC, Callans DJ, et al. Impact of body mass index on safety and efficacy of the subcutaneous Implantable cardioverter-defibrillator. JACC Clin Electrophysiol. 2018;4:652-9.
22. KerberRE, GrayzelJ,HoytR, Marcus M,KennedyJ.Transthoracic resistance in human defibrillation. Influence of body weight, chest size, serial shocks, paddle size and paddle contact pressure. Circulation. 1981;63:676-82.

23. Do K, Chang P, Konecny T, et al. Predictors of elevated defibrillation threshold with the subcutaneous Implantable cardioverter-defibrillator. J Innov Cardiac Rhythm. 2017;8:2920-9.

24. Friedman DJ, Parzynski CS, Varosy PD, et al. Trends and in-hospital outcomes associated with adoption of the subcutaneous Implantable cardioverter defibrillator in the United States. JAMA Cardiol. 2016;1:900-11.

25. Al-Ghamdi B, Shafquat A, Alruwaili N, et al. Subcutaneous implantable cardioverter defibrillators implantation without defibrillation threshold testing: a single center experience. Cardiol Res. 2017;8:319-26.

26. Maurizi N, Tanini I, Olivotto I, et al. Effectiveness of subcutaneous implantable cardioverter-defibrillator testing in patients with hypertrophic cardiomyopathy. Int J Cardiol. 2017;231:115-9.

27. Peddareddy L, Merchant FM, Leon AR, et al. Effect of defibrillation threshold testing on effectiveness of the subcutaneous implantable cardioverter defibrillator. Pacing Clin Electrophysiol. 2018; https://doi.org/10.1111/pace. 13416.

28. le Polain de Waroux JB, Ploux S, Mondoly P, et al. Defibrillation testing is mandatory in patients with subcutaneous implantable cardioverter-defibrillator to confirm appropriate ventricular fibrillation detection. Heart Rhythm. 2018;15:642-50.

29. Baalman SWE, Kooiman KM, Quast A-FBE, et al. Noise caused by involuntary muscle response may inhibit detection of ventricular fibrillation during defibrillation testing. HeartRhythm Case Rep. 2019;6:126-30.

30. Frommeyer G, Zumhagen S, Dechering DG, et al. Intraoperative defibrillation testing of subcutaneous implantable cardioverter-defibrillator systems-A simple issue? J Am HeartAssoc. 2016;5:e3181.

31. Boersma L, Barr C, Knops R, et al. Implant and midterm outcomes of the subcutaneous Implantable cardioverterdDefibrillator registry: the EFFORTLESS study. J Am Coll Cardiol. 2017;70:830-41.

32. Boersma LV, El-Chami MF, Bongiorni MG, et al. Understanding outcomes with the EMBLEM S-ICD in primary prevention patients with low EF study (UNTOUCHED): clinical characteristics and perioperative results. Heart Rhythm. 2019;16:1636-44.

33. Gold MR, Aasbo JD, El-Chami MF, et al. Subcutaneous implantable cardioverter-defibrillator post-approval study: clinical characteristics and perioperative results. Heart Rhythm. 2017;14:1456-63.

34. Weiss R, Knight BP, Gold MR, et al. Safety and efficacy of a totally subcutaneous implantable-cardioverter defibrillator. Circulation. 2013;128:944-53.

35. Quast ABE, Baalman SWE, Brouwer TF, et al. A novel tool to evaluate the implant position and predict defibrillation success of the subcutaneous implantable cardioverterdefibrillator: The PRAETORIAN score. Heart Rhythm. 2019;16:403-10.

36. Quast ABE, Baalman SWE, Betts TR, et al. Rationale and design of the PRAETORIAN-DFT trial: A prospective randomized CompArative trial of SubcutanEous ImplanTable CardiOverter-DefibrillatoR ImplANtation with and without DeFibrillation testing. Am HeartJ. 2019;214:167-74. 